\title{
Photon Gas
}

\author{
A. S. Belyaev \\ Department of Physicotechnical, Joint-Stock Company "All-Russia Thermal Engineering Institute" (JSC "VTI"), Russian Federation
}

Copyright $(2016$ by authors, all rights reserved. Authors agree that this article remains permanently open access under the terms of the Creative Commons Attribution License 4.0 International License

\begin{abstract}
The article suggests that free photon gas is not a thermodynamical system, that is why it can't cool down. Correspondingly, cosmic microwave background cannot be relict. The inflationary cosmic model is based on the previous assumption. That is why the theory of cosmological inflation is not correct.
\end{abstract}

Keywords Statistical Physics, Cosmic Background Radiation, Inflationary Cosmological Model

\section{Introduction}

Statistical physics studies the characteristics of systems, the states of which remain unchanged over time. This state is referred to as an equilibrium. If the system in question can exchange not only energy but also particles with its surrounding environment then its equilibrium, described by Gibbs distribution, does not only depend on its statistical temperature but also on partial potential, sometimes called chemical potential. Processes that take place as a result of a change in states of equilibrium are studied by physical kinetics. However, it is very important that in the framework of physical kinetics transient processes can only be modeled when the system in question, at its final transition points, reaches states of equilibrium. Nonlinear thermodynamics, which can study the stable stationary states of the system that are far from equilibrium is a completely different science and has no relation to the subject under discussion.

Electromagnetic radiation that fills the closed cavity and is in equilibrium with the material forming the cavity, is well described by statistical physics. However, existing statistical laws for electromagnetic radiation in the closed cavity, begin to also characterize, due to historical circumstances, free electromagnetic radiation that is not in equilibrium with the substance and not limited to any real border with the environment. In this paper we consider the consequences of such negligence.

\section{Current Situation}

If an isolated closed system consists of several elements, then, knowing physical parameters of each element at the initial point of time (for example, momentums and coordinates) and laws of interaction going inside the system, one can define physical properties of all the elements for any following moment of time. However, with growth of the amount of elements, the amount of solvable equations becomes so high that their joint solving becomes impossible. But if the laws of interaction between the elements are one-type for all the elements and universal for them, then laws of statistics take effect, in the presence of which we don't have to solve a set of many equations for any individual element, or introduce initial conditions for each individual element, but operate parameters averaged for the considered system, perform statistical averaging. Such systems, for which laws of statistics are valid, are called thermodynamical. Laws of statistics begin working only for systems with a big amount of elements.

Laws of statistics are based on probability theory, but they characterize quite ordinary elements of thermodynamical system, submitting to classical mechanics. Quantum effects bring in some peculiarities in the description of the system, but do not define the final result. After mental eliminating of a small volume from the volume of thermodynamical system, for the elements of such subsystem there can be defined probabilities to have certain coordinates and momentums with infinitesimal deviations from these values, i.e. functions of probability are made for the subsystem of the thermodynamical system of possible magnitudes of coordinates and momentums of its elements which are called functions of statistical distribution. Average values of statistical functions in their essence are equal to time averaging and give the presumable result without tracking real changes of coordinates or momentums of elements of the system, but are defined by the probability theory. But probabilistic nature of results of statistics lies not in probabilistic nature of the elements of the system, but in insufficient amount of initial data (for strict description of the results of interaction of the system elements, the initial parameters of each element are necessary).

An important distinctive feature of a system, for which it becomes possible to consider applicability or inapplicability of laws of statistics, is the absence of decay in the interaction of the elements of the system between each other. In a 
thermodynamical system, the interactions described by it are always non-decaying. The result of non-decay of interactions is the fact that the equilibrium of an isolated thermodynamical system is reached only with leveling of density of observable interactions in the space taken by the system. Correspondingly, with certain parameters of the environment and characteristics of the boundaries, the thermodynamical system can be characterized only by one equilibrium state.

Sometimes the boundary or another system contacting through the boundary with the first one, creates conditions for the existence of the system. For example, in absolutely black body with unchanging temperature, which is a spherical shell, two systems contact with each other: photon gas and matter. Besides, it is rightful to call both systems thermodynamical. At the same time, an absolutely black body and electromagnetic radiation with Planck's spectrum are in thermodynamical equilibrium. But if the substance is suddenly eliminated (the "shell" of absolutely black body is liquidated), then photon gas will no longer represent not only a thermodynamical system, but the system as itself, as photons, without noticing each other, will move away from the initial volume of the thermodynamical system "photon gas" to infinite distances (the system will appear to be unstable and decaying).

Substance can emit and absorb radiation. All kinds of luminescence (chemiluminescence, photoluminescence and others) are always non-equilibrium. Only heat radiation can be equilibrium, when the spectrum of radiation stays unchanged arbitrarily long time. The amount of energy radiated by a material body in condition of equilibrium is strictly equal to the amount of the absorbed energy and is defined by the temperature of the body. If material bodies are surrounded with impenetrable shell with ideally reflecting interior surface and vacuumize its interior volume, then after a definite period of time all the bodies will have the same temperature. Between material bodies and heat radiation there will be equilibrium. And to equilibrium states and processes, laws of thermodynamics can be applied. It is a convention that equilibrium heat radiation is called photon gas. Thus, photon gas can be described by thermodynamical laws. For example the adiabatic exponent for photons is $\gamma=$ $4 / 3$. But photon gas is a gas only as long as it is in equilibrium with substance.

Distribution of molecules of ideal gas in different conditions is described by Boltzmann distribution. A system of molecules with antisymmetrical wave functions, submitting to Pauli principle, is described by Fermi's statistics. A system of molecules with symmetrical wave functions is described by Bose's statistics. If electromagnetic radiation is in equilibrium with the substance, then Bose's statistics is applicable to it. An atom or molecule emitting electromagnetic radiation, changes its mechanical angular momentum to that carried by the photon that is moving away from it, the magnitude of which is a multiple of the reduced Planck constant $\hbar$ (angular momentum, expressed in units of $\hbar$, is an integer).
Photons do not interact with each other, except for the non-linear light waveguides. The creation of electron-positron pairs during the absorption of gamma-ray in a strong non-uniform electric field is due to the collision of gamma rays with high-energy virtual photons in order to maintain the validity of the law of conservation of momentum. But this is a theoretical assumption. In practice, elastic or non-elastic photon collisions have not been observed, as photons are transparent to each other at the intersection of their trajectories. However, as long as photon gas is in equilibrium with the substance, it can be described by statistics. Yet, it brings in a specific peculiarity into the description of photon gas: the amount of photons in a photon cloud is not a constant and is defined by conditions of heat equilibrium. Such peculiarity, while taking into consideration minimal free gas energy, automatically leads the chemical potential of the photon gas to zero. That is why the photons distribution in different quantum states turns out to be Planck distribution. In view of the absence of photons' interaction with each other, photon gas is considered to be ideal.

Photons also have other features. For example, the speed of particles that have no rest mass is always constant and equal to the speed of light $c$ which is related to their energy $\varepsilon$ and momentum $p$ by $\varepsilon=p c$. Moreover, different photons have their own individual values of energy and momentum, depending on the frequency of electromagnetic radiation. Another feature is that photons can change the direction of motion only by reflection from the boundary separating the photon gas from the surrounding environment. If any material body is within the photon gas filled cavity, it will absorb and emit photons. In the state of equilibrium of the system the total energy of the absorbed and emitted body of photons has to be equal, but in a separate act the frequencies of absorbed and induced electromagnetic radiation might not match.

Gamow, developing the Big Bang model, showed, and quite rightly, that at thermodynamical equilibrium, photon gas in plasma has Planck spectrum. After recombination of a plasma cloud, photon gas, being in equilibrium with plasma, has to be released. But further he supposed, and all the scientific society followed him, that the released photon gas remains a gas and cools down. Gamow determined the approximate temperature of this gas for today and predicted its existence in the form of cosmic background. The theory of inflation did not exist at that moment.

After Hubble's discovery, which proves the reality of expanding of the available nowadays to our observations Universe matter, and Penzias and Wilson's monitoring, that discovered background equilibrium electromagnetic radiation with Planck's spectrum, the average density of energy of which, despite low efficient temperature, significantly exceeds the density of energy of stars radiation in the interstellar space, the version of the marked out moment of time, connected with the birth of the Universe and the beginning of its expansion from an ultratight and ultrahot space region, became generally accepted (as we 
observe the Planck's spectrum, the radiation can't be connected with active annihilation processes etc).

Within the bounds of the cosmological model "Big Bang", our Universe appeared from a singular point. The expanding cloud of matter cooled down, in a certain time plasma was formed, then gas, and after that galaxies were formed out of gas. But in a reverse chronology, our Universe does not roll into a point (Planck length cannot be achieved). That is why the inflationary model appeared, specifying the standard of the Big Bang model. Inflation is the process of accelerated expanding of a local perturbation in an even scalar field, which is characterized by a high density of energy. The assumed physical processes, occurring at the stage of birth of the Universe, can be examined in $[1,2]$.

According to the inflationary model, the birth of the Universe is not connected with the appearance of a singular point, but it was marked by forming space-time and its unfolding by exponential law with superluminal velocity. Matter formation began in $10^{-35} \mathrm{~s}$ : oscillations with generating matter began to get excited along the entire stretched zone, not in a point. In the course of time this model included new concepts of "dark matter" and "dark energy". Like in the standard model, the generated matter goes through plasma and gas state, but after recombination of plasma, first not stars are formed, but straight away galaxies without stars, and not out of matter, but out of dark matter. After that, formation of stars and galaxies appears like in the standard model, but, firstly, with formation of black holes, and, secondly, with appearance of the second phase of inflationary process about 6.5 billion years ago under the influence of dark energy, which was connected with accelerated recession of galaxies. Homogeneity, isotropy and euclidity of space are the consequence of stretching of the local by many orders. Heterogeneities, because of which the galaxies were formed, are the result of stretching of quantum fluctuations, which were occurring in the moment of the birth of the Universe.

The inflationary model allowed explaining, without resorting to the notion of singularity, why at the birth of the Universe an ample quantity of elementary particles in a small amount of space could be generated. With appearance of new knowledge and discoveries, the model was constantly specified, and today it is called "The coordinated inflationary Lambda CDM cosmological model". In the inflationary theory it is presumed that the initial quantum fluctuations of vacuum of a size $10^{-29} \mathrm{~m}$ were stretched by many orders of magnitude and had to cause heterogeneities of spectral power of relict electromagnetic radiation. The discovered Gaussian distribution of temperature heterogeneities of the relict radiation confirmed the inflationary theory. The measuring results obtained by the BISEP telescope in 2010-2012, which is located in the South Pole, revealed the polarization of relict microwave electromagnetic radiation, which can be explained by relict gravitational waves. The appearance of relict gravitational waves results from the inflationary theory. The announcement of it appeared on 2014 March 17. Nevertheless, the concordance of the obtained results with the measuring results of satellites WMAP and Planck is not achieved yet.

Thus, today it is commonly accepted that microwave background is relict, and inflationary cosmological model holds a dominating position.

\section{Analysis}

But while photons' colliding, they don't interchange impulses and energy, they show themselves as waves. That is why, from clearly physical point of view, it is impossible to talk about cooling down of the gas consisting of the particles not interacting with each other. While expanding of photon "gas" its density will change, but not its temperature, which, in accordance with traditional interpretation, is defined as the average energy of massless particles of the considered gas. It is possible to talk about cooling down of photon gas, being in equilibrium with matter, as the spectral density of radiation power of an absolutely black object depends on its temperature. But free photon gas is not able to cool down, because free massless photons can't change their energy without absorbing and re-radiation with the matter atoms. For photons, isolated from the matter, there is no notion of "the function of statistical distribution" in phase space of coordinates and impulses, i.e. statistical laws are not applicable for them. We cannot affirm that an infinitesimal subsystem during a long period of time goes through all possible values of the phase space. That is why we cannot describe photon gas without knowing the initial conditions for each photon of the system. Correspondingly, we have no right to say, that photon gas, not being in equilibrium with the matter, is subject to some kind of statistics. No scientist of the world could ever try to explain the selection of photons in the Universal background, which interact with each other, but don't interact with all the other photons of the Universe from gamma rays to radio waves, the total density of which doesn't yield to the photons density of the Universal background. Nor could he elucidate, why the cooling down photon gas of the Universal background doesn't become single-frequency, but keeps its Planck looks, complying with the Wien displacement law.

It is also necessary to take into consideration that although the process of plasma recombination in astronomical standards didn't continue for a long time, was almost immediate, still by its nature it is not single-stage. That is why the recombination was smooth and it gradually released the electromagnetic radiation with a larger density of photons, but with their smaller temperature. Even by this reason it is difficult to accept the electromagnetic microwave background radiation to be relict.

Moreover, taking into consideration the fact that the Universal expansion after photons release exceeds by many orders of magnitude the time of the connected expansion, when the radiation and the matter were in thermodynamical equilibrium, we simple would not be able to fixate equilibrium Universal background: absolutely every single 
photon of the past (born before their release of the matter from captivity) are obliged to run away from us (created from the matter, which was once released by radiation) for boundless distances, run away not by the laws of gas expansion, but by rectilinear trajectories with the velocity of light.

It would be useful to pay attention to the discrepancy of the densities of mass and radiation, which we could try to explain by the intensive annihilation processes of matter and antimatter at the stage of released radiation, but it doesn't combine well with the existing theory of the Big Bang. The point is that the mass density of massless particles (calculated from their energies) at the early stages of the Universe expansion had to exceed significantly the density of the matter both in mass, and in the quantity of particles. But the modern observations show that the mass density of protons exceeds the mass density of photons approximately by 2000 times.

The inflation model of the birth of the universe appeared after the recognition of the cosmological background radiation as a relic radiation. And the reason for the creation of the inflation model has nothing to do with low energy photons of background radiation. It is unacceptable to consider the contribution of space-time expanding into decrease of a photon gas temperature, as the displacement of waves' lengths into the red area at the expense of expansion will occur without complying with the Wien displacement law. Photon gas has always been considered to be a thermodynamic system. That's why the inflationary model cannot explain the low energy of photons evenly distributed in the volume of the cosmic background, and it never had such purpose. Nor can it remove the problem of the possibility of the relict radiation registration by the Earth observer. But, what is the most important, in spite of the progress of the inflationary model, it generates many new uncertainties and essences: it is not certain what the physical essence of the initial scalar field is, the physical fullness of the initial quantum fluctuations, the evolution of vacuum, the going processes and the space zone of matter generation, the principle of inertia, connected with the matter expansion after inflation, and others.

Recently it has often been affirmed that background electromagnetic radiation is the remains of electromagnetic radiation of the first Planck seconds after the birth of the Universe. But such models only generate new insoluble issues, issues of principle. First of all, it is connected with explaining the origin of electromagnetic radiation with Planck spectrum before the matter formation. That is why it is inadmissible to withdraw problems with red displacement that way.

\section{Results}

Thus, the universal background cannot be relict. Only matter can be the source of it; the low-temperature matter, not having a high velocity relative to an Earth observer. Such characteristics of the background source are accepted because Doppler displacement does not provide in the distribution of the radiation power the displacement of peak into long-wave area from the hot matter, moving off with high velocity.

\section{Discussion}

The fact that the microwave radiation originates from the depths of space is experimentally proven by measurements from the paired telescope Keck located in Hawaii. This observation confirms that the main matter density is concentrated at the periphery of the Universe. This should be a basic presupposition of a new theory of the Universal background.

The equilibrium of background radiation is connected with uniform matter distribution on the fringes of the Universe. The homogeneity of the averaged characteristics of the massive halo of the Universe is connected with spacial radial symmetry of matter distribution in the process of birth of the Universe, despite the presence of galaxies and the absence of observed antimatter. The source of universal background can be continuous spherical homogeneous upon average gas layers with sufficient optical thickness for formation of the Planck's spectrum near the outer periphery matter or other formations and structures at the periphery of the Universe, consisting of matter.

The existence of the massive Universal halo is confirmed by isotropic background roentgen radiation, the density of which is less than the density of "relict" microwave radiation. Another source of roentgen background radiation, apart from the periphery zones of the Universe, are difficult to imagine even theoretically (possible versions of the roentgen background origin exist, but all of them are quite unconvincing). After this important discovery the scientists also admitted the existence of a massive halo universe, but over time, this approach was forgotten. Based on the new understanding of the nature of the background microwave radiation it seems useful to return to the hypothesis of the existence of a massive halo universe.

Massive halo of the Universe can both run away from us and approach to us. Taking into account the existence of two kinds of background, the velocity of movement of the Earth in relation to the Universal halo is moderate. It is absolutely evident that the Universal halo consists of hidden mass. The phrase "hidden mass" doesn't mean accumulation of common cold matter or, especially, dark matter of unknown nature. For example a neutron star, without matter accretion, is not a source of electromagnetic radiation. Concrete models of structure of the periphery zones of the Universe and the cosmological picture of the world are a far way off. The question of the possibility of formation of a massive Universal halo does not cause any inherent difficulties within the boundaries of the existing scientific knowledge. However, considering possible schemes of the Universal evolution is a separate topic for discussion. 


\section{Conclusions}

The main conclusion from this analysis is part of statistical physics and is as follows:

- free photon gas is not a thermodynamical system and it cannot cool down.

This conclusion is the key to cosmology, leading to the need for revision of its foundations. Implications of the findings are as follows:

- cosmic microwave background cannot be relict;

- the inflationary cosmological model is not correct;

- the standard cosmological model needs a profound modification;
- the Universe is surrounded by a massive halo.

\section{REFERENCES}

[1] D. S. Gorbunov, V. A. Rubakov. Introduction to the Theory of the Early Universe: Hot Big Bang Theory, World Scientific Publishing Company, 2011.

[2] D. S. Gorbunov, V. A. Rubakov. Introduction to the Theory of the Early Universe: Cosmological Perturbations and Inflationary Theory, World Scientific, Singapore, 2011. 